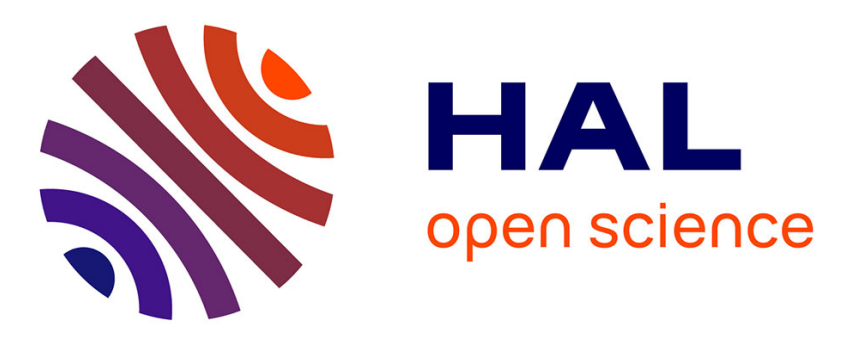

\title{
Preliminary survey of backdrivable linear actuators for humanoid robots
}

\author{
Philippe Lucidarme, Nicolas Delanoue, Franck Mercier, Yannick Aoustin, \\ Christine Chevallereau, Philippe Wenger
}

\section{- To cite this version:}

Philippe Lucidarme, Nicolas Delanoue, Franck Mercier, Yannick Aoustin, Christine Chevallereau, et al.. Preliminary survey of backdrivable linear actuators for humanoid robots. 22nd CISM IFToMM Symposium on Robot Design, Dynamics and Control (ROMANSY'2018), Jun 2018, Rennes, France. pp.304-313, 10.1007/978-3-319-78963-7_39 . hal-01989982

\section{HAL Id: hal-01989982 \\ https://hal.science/hal-01989982}

Submitted on 22 Jan 2019

HAL is a multi-disciplinary open access archive for the deposit and dissemination of scientific research documents, whether they are published or not. The documents may come from teaching and research institutions in France or abroad, or from public or private research centers.
L'archive ouverte pluridisciplinaire HAL, est destinée au dépôt et à la diffusion de documents scientifiques de niveau recherche, publiés ou non, émanant des établissements d'enseignement et de recherche français ou étrangers, des laboratoires publics ou privés. 


\title{
Preliminary survey of backdrivable linear actuators for humanoid robots
}

\author{
Philippe Lucidarme ${ }^{1}$, Nicolas Delanoue ${ }^{1}$, Franck Mercier ${ }^{1}$, Yannick Aoustin ${ }^{2}$, \\ Christine Chevallereau ${ }^{2}$, and Philippe Wenger ${ }^{2}$ \\ 1 LARIS, 62 avenue Notre Dame du Lac, 49000 Angers, France, \\ philippe.lucidarme@univ-angers.fr \\ 2 LS2N, UMR CNRS 6004, 1 Rue de la Noë, 44300 Nantes, France.
}

\begin{abstract}
This paper presents a preliminary survey of the use of direct drive linear motors for joint actuation of a humanoid robot. Their prime asset relies on backdrivability, a significant feature to properly cushion high impacts between feet and ground during dynamic walking or running. Our long-term goal is the design of high performance human size bipedal walking robots. However, this paper focuses on a preliminary feasibility study: the design and experimentation of a mono-actuator lower limb.
\end{abstract}

Keywords: biped robot, linear actuator, backdrivability.

\section{Introduction}

During the last decades, several research teams and companies have developed humanoid robots. The evolution of this field has known rapid progress. The robotics contest DARPA Challenge is a great illustration of the current state of the art [4]. However, everyone will admit that humanoid robotics is still at an early stage. Many scientific obstacles remain. Despite the numerous existing bipedal robots, dynamic walking, running, or jumping are still facing technical and scientific barriers.

Historically, humanoid robot architectures have inherited from industrial robots. Rotary actuators transmit motion to the robot joint via gear reducers. Most designers have converged on a combination of brushless DC motors and Harmonic Drives [7]. The gear ratio manages the balance between torque and speed, but generally prevents the actuator from being backdrivable. Backdrivability is the ability for bidirectional interactive transmission of force between input axis and output axis [3]. An example of this limitation is the inability of humanoid robot to properly cushion high impacts between feet and ground. Although the human body has almost no passive shock absorbers in its anatomy, human beings can deal with these impacts since muscles play the role of active shock absorbers $[2,9]$. This is only possible because the joints and muscles are backdrivable, otherwise joints would suffer from repeated traumas. This analysis is transposable to humanoid robots and backdrivability is a prior challenge to 
overcome. The pioneers of artificial legged locomotion were facing this issue and several solutions have been proposed. A selection of related works is presented in the first section. Sections 4 and 5 of the paper are dedicated to the optimal dimensional geometry calculated through numerical optimization. Section 6 introduces an in-house proof of concept and the experimental results are discussed in section 7. To end this paper, an overall conclusion discusses about the encouraging results on shock absorption during impacts and suggest unmatched future performances for humanoid robots.

\section{Related works}

This section presents previous works that explored the design of backdrivable actuators for humanoid robotics. A non-exhaustive selection of solutions is discussed here: pneumatic, hydraulic, cables, and serial elastic actuators (SEA).

Pneumatic actuators were experimented with interesting results, but are irrelevant for high torque joints and the use of compressors is an obstacle for embedded system [12].

Hydraulic actuator is the solution favored by Boston Dynamics [11,4]). Well known for its performance in rough terrain, this is a noisy, expensive and heavy to implement option.

Cables actuators has been experimented on the Sherpa robot [10]. To the best of our knowledge, these robots have not yet demonstrated satisfying performances.

The Virginia Tech robotics laboratory designed an advanced linear serial elastic actuator embedded in three generations of robots: SAFFIR, THOR and ESCHER [5]. These works are the starting point of the survey presented in this paper. Indeed, the team shown that linear motors could be a well-suited solution for the design of humanoid robots. Size of the actuator is no longer limited by the joints area and can be placed into the links. Mechanical architecture can easily be inspired by human anatomy where actuators replace muscles. Unfortunately, SEAs were designed around ball screw drives and backdrivability is disputable. Relying on these previous works we investigated the use of electric direct drive linear motors for joints actuation. This actuator is presented in the next section.

\section{Direct drive linear actuators}

Direct drive linear motors must be distinguished from mechanical linear motors. These latter embed a rotary actuator combined with a mechanical transformation (belt or screw drives). The mode of operation of the direct drive motors discussed in this paper is as a Lorentz-type actuator. The linear motor consists of two parts: the slider and the stator. The slider is made of neodymium magnets that are mounted in a high-precision stainless steel tube. The stator contains the motor windings, bearings for the slider, position capture sensors, and a microprocessor circuit for monitoring the motor. An illustration of the motor used in 
our experiments can be found in Figure 1. To avoid overcomplicating the following sections, the term "linear motor" necessary implies "direct drive linear motors", unless otherwise specified.

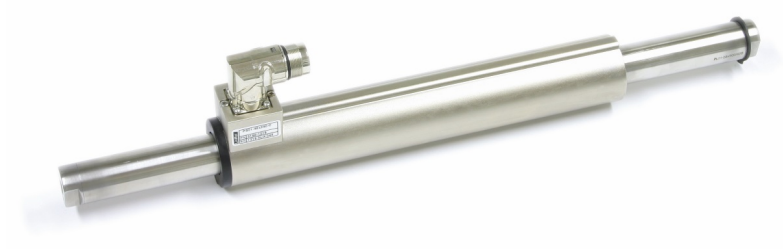

Fig. 1. Photography of direct drive linear actuator (source LinMot@).

In the absence of any movement transformation device (unlike belt or screw drives) the slippage is limited to the friction between the stator and the slider. When unpowered, the force opposed to the motion of the slider is composed of the previous friction added to the magnetic strength between magnets and windings. In practice, this countervailing force is negligible compared to the maximum peak force the motor can produced when powered. This is the reason we consider this actuator as being highly backdrivable. The question addressed in this paper can be summarized as the following: are linear actuators well-suited for actuating humanoid robots ? Underlying issues remain behind this general question:

- Are such actuators powerful enough to raise a human-size robot?

- Can such architecture deal with high impacts between feet and ground?

- How should we design the controller to manage the balance between joint positioning and backdrivability during impacts?

\section{Methods}

The most commonly used method for designing humanoid robots is an openended cyclic methodology [5]. Link properties are derived from a detailed CAD model after each design milestone and testing in simulation to iteratively refine joint specifications. Since this method is time consuming and generally not efficient to successfully build a robot right the first time (Figure 2 right), we decided to start with a refined proof of concept. The latest is composed of three joints located in the same plane (see Figure 2 left). The hip and the ankle are passive while a linear motor actuates the knee. As a single actuator is not sufficient to ensure the standing of the system, a gantry constrains the position of the hip and ankle on a vertical line, thanks to a slider rail as detailed in Figure 5. The simplicity helps focusing on the key points of our research: backdrivability, shock absorption and optimal design. 

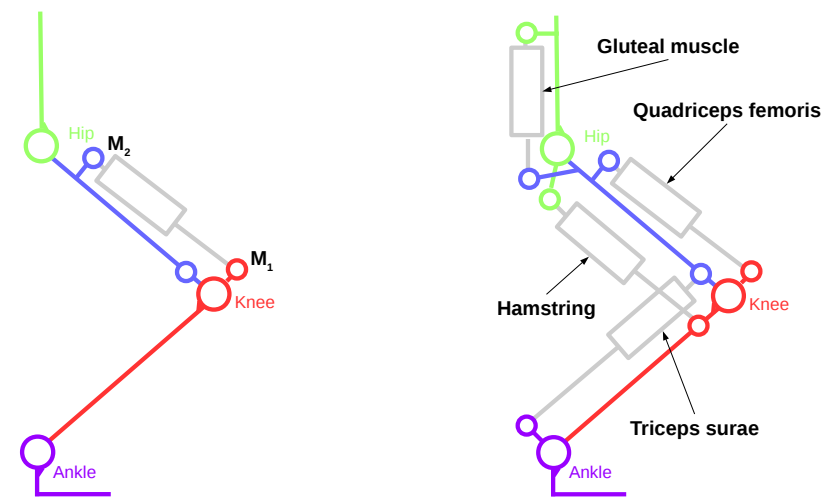

Fig. 2. The single actuator architecture studied for this preliminary work (left). A candidate bi-articular architecture for a future $2 \mathrm{D}$ walking robot. (right)

The first step of our method is the geometrical design of the proof of concept. Behind simplicity lies the question of optimal motor positioning. The actuation system is equivalent to a connecting rod/crank and a close attention must be paid to singularities. Preliminary calculation shown that the fixation points of the motor highly influence the global performance of the system. A numerical optimization algorithm is used in order to find the optimal motor position. An analytic modeling was initially considered to optimize the geometry. This approach has proven difficult, mainly due to the non-linear profile of the actuators. The analytical approach would also be impossible to extend to more complex robots, which is our long-term goal. In light of this conclusions, we switch to numerical optimization methods which can handle more complex architectures.

\section{Numerical optimization}

To facilitate the understanding of the following, some definitions deserve to be clarified. An architecture is a kinematic chain describing the strategic placement of motors. Figure 2 shows examples of architecture. A geometry is a specific positioning of the motors. In this work, we plan to optimize the placement of a motor for a given architecture. The result of this optimization is - ideally the optimal geometry. A configuration is a given posture of the robot (like for industrial robotics). The concepts of articular or singular configuration are also similar.

To simplify this preliminary study, an architecture that does not use several actuators has been chosen (See Figure 2 left). As the quadricep femoris is the most powerful muscle in the human anatomy, it seems relevant to study the architecture for which the single actuator motor is placed at the location of this muscle. Indeed, we hope that obtained results could be extended to more complicated architectures, that is to say with several actuators. 

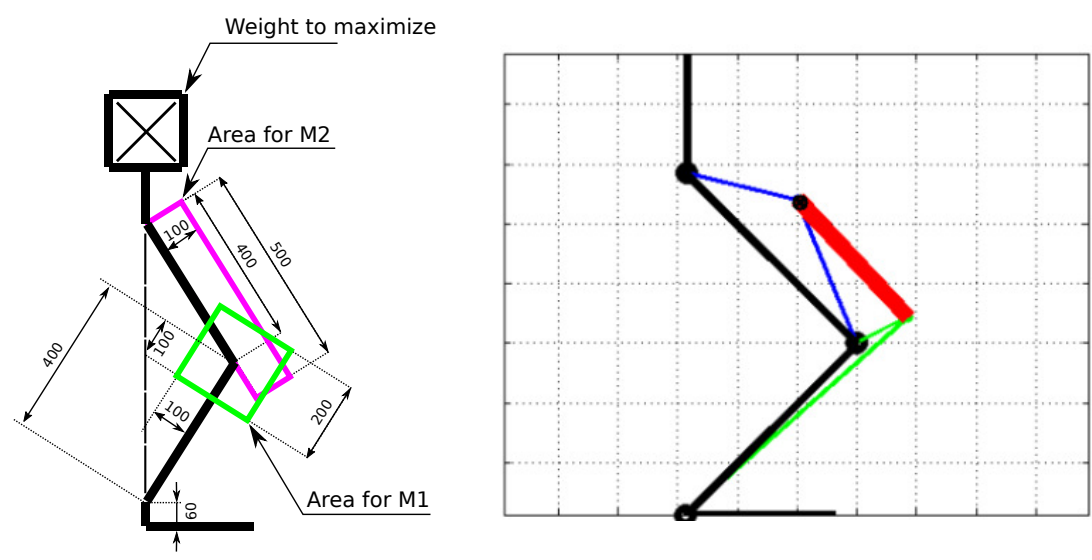

Fig. 3. Left: parameters of the model and restricted areas for $M_{1}$ (green) and $M_{2}$ (purple). Right: optimized position of the motor (red).

Hypothesis: in the following, we will assume the architecture to optimize is given in Figure 2 left. The parameters (link lengths) of our model have been selected based on previous works on human anatomy and body segment parameters [1], [13], and [8]. These parameters have been scaled to a $1.6 \mathrm{~m}$ tall humanoid robot. The criterion to define an optimal design of our system is summarized by:

$$
\max _{M_{1}, M_{2}} \min _{\alpha} W\left(\alpha, M_{1}, M_{2}\right)
$$

where:

- $W$ is the weight supported by the trunk, i.e. scalar maximized by the optimizer.

- $M_{1}$ is the coordinates of the lower fixation point of the motor (attached to the leg and restricted to the green area in Figure 3)

- $M_{2}$ is the coordinates of the higher fixation point of the motor (attached to the thigh and restricted to the purple area in Figure 3)

$-\alpha$ is the knee angle in the range 0 (full knee extension) to 135 degrees with a step of 0.7 degrees.

Since we expect an anthropomorphic geometry, we added constraints on location of points $M_{1}$ and $M_{2}$. This explains the restricted area illustrated in Figure 3. Our goal is to calculate the optimal coordinates of $M_{1}$ and $M_{2}$ to maximize the weight supported by the trunk.

Optimization has been performed and compared with seven algorithms (Monte Carlo, Genetic algorithm, non linear programming solver, simulated annealing, pattern search, Nelder \& Mead, constrained Nelder \& Mead [6]). To avoid getting stuck in local minima, each algorithm was run thousand times with random initial conditions. Results of best geometry and average over the thousand runs are illustrated in Figure 4. 


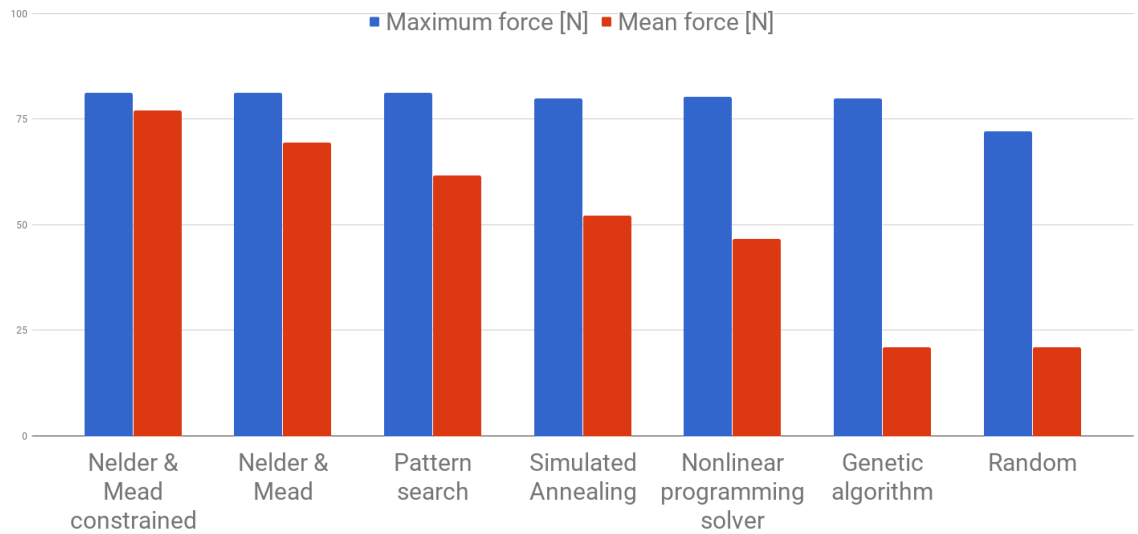

Fig. 4. Comparison of optimization algorithms

The optimal geometry is illustrated in Figure 3 right. This solution lies on the boundary of restricted area, a result that is in accordance with the analytic preliminary study. The maximum weight of $81.2 \mathrm{~N}$ is not enough for a human-size robot, but redundancy and the addition of bi-articular motors will increase the performances for future prototypes.

This survey highlights that most algorithms converge - at least once - to a near-optimal global solution. Analyzing the mean and detailed results shows that Nelder \& Mead constrained is the less sensible to initial conditions. More simulations have been performed for which results are not detailed here. Therefore, these additional optimizations confirm that about 10 runs are sufficient to converge to the optimal geometry with Nelder \& Mead constrained. Note that this comparison is of primary interest to extend this work to a further bi-articular robot geometry optimization as illustrated in Figure 2 right. As a conclusion, it may be noted that these numerical methods have reasonable convergence times and that this optimization technique as well as the general approach could be used in the 3D case.

\section{Experimental setup}

Based on the previous optimization results, a proof of concept has been designed. The CAD model is presented in Figure 5.

Actuator and drive are manufactured by LinMot. Detailed specifications can be found on the LinMot website with the following hardware reference:

- Slider: PL01-28x410/330 - 28mm, lg=410mm

- Stator: PS01-48x240-C

- Drive: C1100-GP-XC-1S-000 (72V/25A) 


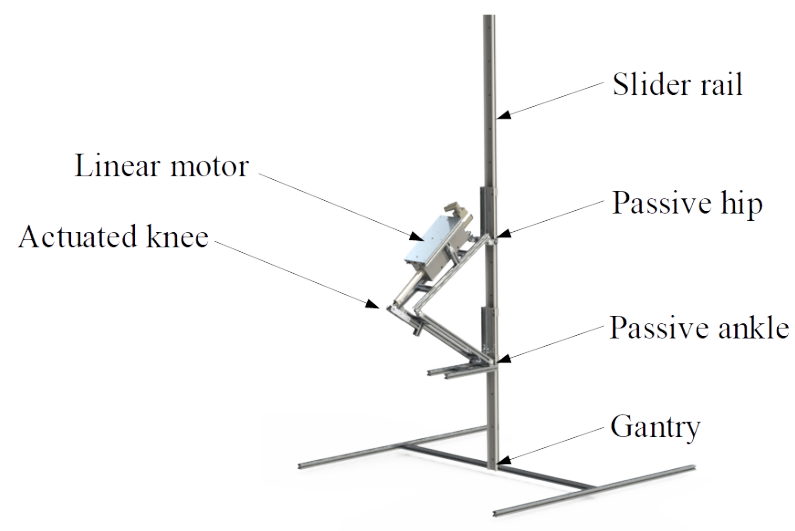

Fig. 5. CAD model of the experimental setup.

The C1100 drive includes a PID position controller with embedded current/force limiter. We added a force controller on top of the PID controller. The whole system acts as a tunable spring where the inputs are the spring stiffness $(\mathrm{K})$ and the spring nominal position (Xsetpoint). A schematic overview of the global architecture is presented in Figure 6. Note that the total weight of the whole falling apparatus is $8.3 \mathrm{Kg}$.

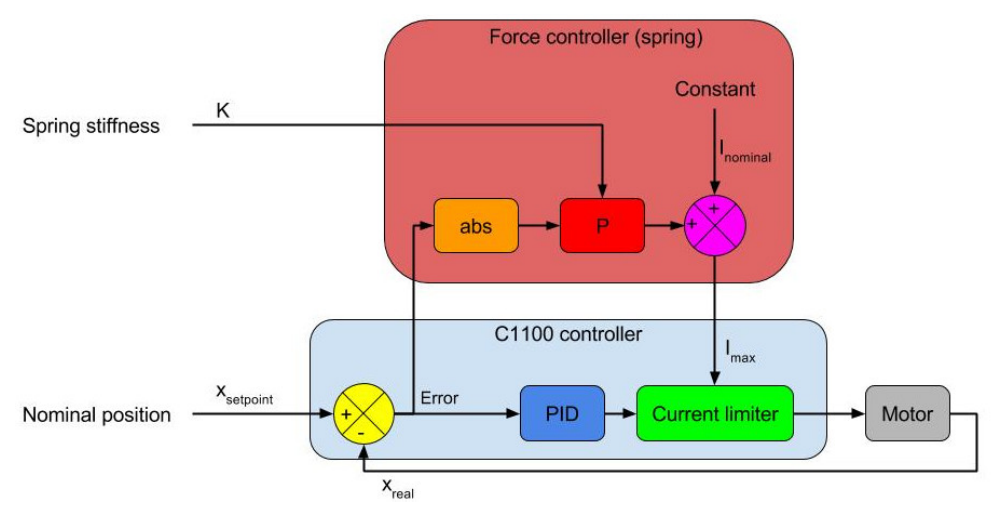

Fig. 6. Schematic overview of the controller.

Two inertial measurement units (IMU) have been attached to the foot and hip to measure accelerations, in order to estimate the efficiency of shock absorption during impact. The two IMU were designed in our laboratory and are based on an Arduino shield with a maximum sampling rate of $1 \mathrm{KHz}$ 


\section{$7 \quad$ Results}

The design of the leg has been done to be able to carry the heavy torso. Now the objective of the experiment is to test the behavior of our simplified leg during a falling/ground impact. The chosen actuator with its backdrivability will be able to properly react to the impact while classical actuators will submit the structure to extensive internal constraints. Several experiments have been performed on cushioned landing of $0.1,0.2,0.3,0.4$ and $0.5 \mathrm{~m}$ high fall (illustrated in Figure 7). The first conclusion is the ability of the system to successfully deal with high impacts. The motion is clearly a flexible and natural cushioning like human beings.

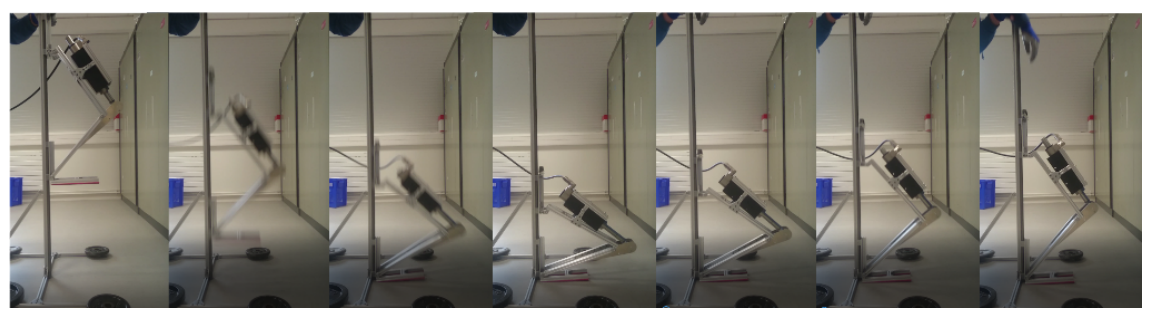

Fig. 7. Experiment on cushioned landing of a $40 \mathrm{~cm}$ high fall. https://youtu.be/RfjsJvsW6WM

Data have been recorded during experiments. The $40 \mathrm{~cm}$ high fall data are plotted in Figure 8. The first graph shows the foot acceleration with a peak at zero second, time where the impact occurs. Note that since the duration of the impact is very short, sensors were not able to catch the maximum value which is much higher than the peak reported in Figure 8. The third graph in Figure 8 is the force produced by the linear actuator, these data were obtained by measuring the consumed current.

The fall is visible between -200 s and 0s where the falling acceleration compensates the gravity: recorded acceleration is null during falling. Analyzing knee angle and motor force shows how the impact is transformed from a short time / high amplitude to a spread over time / smoothed amplitude energy by the mechanism.

\section{Conclusion and perspectives}

In this paper, the backdrivability was exploited for the design of a humanoid lower limb able to cushion very high impacts, which is an original approach, to the best of our knowledge. The knee of the proposed leg is actuated with a linear actuator while the hip and ankle joints are passive. The design was optimized for a prescribed load. A test-bed prototype was built and experimental results 


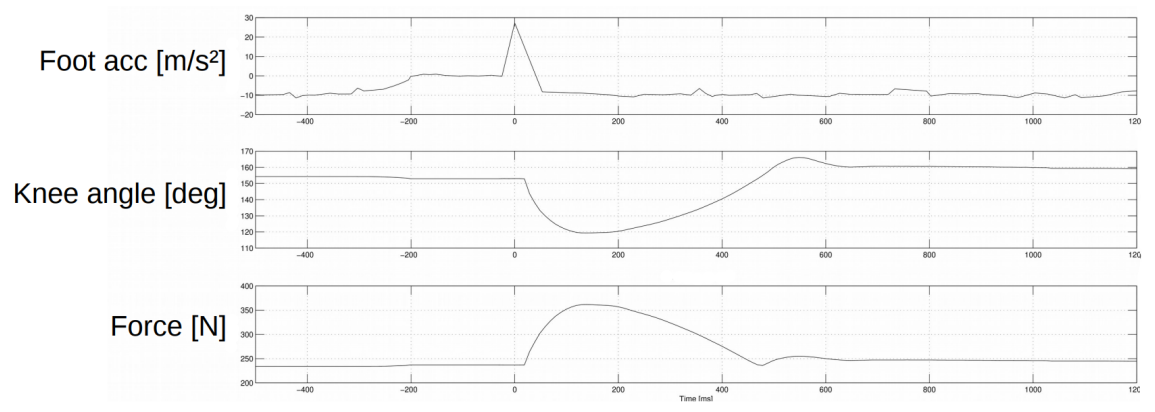

Fig. 8. Foot acceleration, knee angle and motor force before, during and after impact.

demonstrated the ability of the leg to cushion very high impacts between the feet and the ground, showing the central role played by the linear actuator. Future work will investigate the benefits of combining backdrivability and the use of bi-articular joints in the design of a complete humanoid leg.

\section{References}

1. Drillis, R., Contini, R., Bluestein, M.: Body segment parameters. New York University, School of Engineering and Science Research Division, NY (1966)

2. Ferenczi, M.A., Bershitsky, S.Y., Koubassova, N.A., Kopylova, G.V., Fernandez, M., Narayanan, T., Tsaturyan, A.K.: Why muscle is an efficient shock absorber. PLOS ONE 9(1), 1-6 (2014). DOI 10.1371/journal.pone.0085739

3. Ishida, T., Takanishi, A.: A robot actuator development with high backdrivability (2006). DOI 10.1109/RAMECH.2006.252631

4. Johnson, M., Shrewsbury, B., Bertrand, S., Wu, T., Duran, D., Floyd, M., Abeles, P., Stephen, D., Mertins, N., Lesman, A., Carff, J., Rifenburgh, W., Kaveti, P., Straatman, W., Smith, J., Griffioen, M., Layton, B., De Boer, T., Koolen, T., Pratt, J.: Team ihmc's lessons learned from the darpa robotics challenge trials. J. of Field Robotics 32 (2015)

5. Knabe, C., Seminatore, J., Webb, J., Hopkins, M., Furukawa, T., Leonessa, A., Lattimer, B.: Design of a series elastic humanoid for the darpa robotics challenge (2015). DOI 10.1109/HUMANOIDS.2015.7363452

6. Lagarias, J.C., Reeds, J.A., Wright, M.H., Wright, P.E.: Convergence properties of the nelder-mead simplex method in low dimensions. SIAM J. on Optimization 9(1), 112-147 (1998). DOI 10.1137/S1052623496303470

7. Lahr, D.F., Yi, H., Hong, D.W.: Biologically inspired design of a parallel actuated humanoid robot. Advanced Robotics 30(2), 109-118 (2016). DOI 10.1080/01691864.2015.1094408

8. de Leva, P.: Adjustments to zatsiorsky-seluyanov's segment inertia parameters. J. of Biomechanics 29(9), 1223 - 1230 (1996). DOI 10.1016/0021-9290(95)00178-6

9. Minetti, A.E.: Using leg muscles as shock absorbers: theoretical predictions and experimental results of drop landing performance. Ergonomics 41(12), 1771-1791 (1998). DOI 10.1080/001401398185965. PMID: 9857837 
10. Olaru, I.M.C., Krut, S., Pierrot, F.: Novel mechanical design of biped robot sherpa using 2 dof cable differential modular joints (2009). DOI 10.1109/IROS.2009. 5354425

11. Raibert, M.: Bigdog, the rough-terrain quadruped robot (2008)

12. Rosendo, A., Nakatsu, S., Narioka, K., Hosoda, K.: Pneupard: A biomimetic musculoskeletal approach for a feline-inspired quadruped robot (2013). DOI 10.1109/IROS.2013.6696540

13. Zatsiorsky, V., Seluyanov, V.: The mass and inertia characteristics of the main segment of human body. Biomechanics VIII: Proc. of the eighth international congress of biomechanics. Human Kinetics Publishers Champaign Il 4 (1982) 